\title{
Assessment of the Adherence to Covid19 Prevention Protocols By Auxiliary Health Personnel In The Kenya Medical Training College Quarantine Centres
}

\author{
Jacinta Mukonzo $^{a^{*},}$ Marsellah Ogendo ${ }^{a}$, Kelly Oluoch ${ }^{a}$, Egglah Kiplagat $^{a}$, Claris Ambale \\ ${ }^{a}$ Kenya Medical Training College \\ ${ }^{\mathrm{b}}$ Machakos Level 5 Hospital
}

DOI: 10.29322/IJSRP.11.01.2021.p10930

http://dx.doi.org/10.29322/IJSRP.11.01.2021.p10930

\begin{abstract}
Background: The global health crisis from the corona virus disease pandemic is not precedent. Globally, it has been shown that the control of the spread of this disease and other diseases is closely related to the situation of Human Resource for Health in a country. (Figueroa-Munoz et al., 2005).Health personnel include front line workers (clinicians, nurses, pharmacists, public health practitioners, medical laboratory personnel) among others as well as auxiliary personnel to the health care system. Auxiliary health personnel play a pivotal role in prevention of spread of the diseases as well as supporting the functioning of a health care system. These workers can also be vectors of spread if tßheir services are not streamlined and aligned to the preventive measures put in place. The WHO has prescribed measures to be followed to prevent the spread of Covid19.This research sought to determine the extent of implementation of Covid 19 protocols by auxiliary personnel in quarantine centers.

Methodology: An institutional based cross- sectional descriptive study was carried out in all the Kenya Medical Training College campuses with quarantined individuals. Purposive sampling was done. Data was collected using an observation checklist and analysed using SPSS version 22 .

Results: There were cleaning, security and catering personnel in the various quarantine centres. There were varying extends of compliance to Covid19 prevention protocols. Most of the auxiliary health personnel were aware how Covid19 is spread at $98.3 \%$. The most adhered protocol was wearing of masks by security personnel at $100 \%$. The greatest lapse was at $66.7 \%$ by security personnel not guiding visitors to wash hands or use a alcohol based sanitizer. There was a significant lapse by cleaning personnel not wearing PPE while cleaning at $44.4 \%$. Three cleaning staff had already contracted Covid19 by the time of the study.
\end{abstract}

Conclusion and recommendation: There were significant lapses in the adherence to Covid19 protocols by auxiliary health personnel. There is need to focus on auxiliary health personnel in preventing the spread of infections especially in pandemics.

Index Terms- Adherence, Auxiliary, Covid19, Protocols, Quarantine

\section{$T$} 1.1 Background

he global health crisis from the corona virus disease pandemic is un precedented. Globally, it has been shown that the control of the spread of this disease and other diseases is closely related to the situation of Human Resource for Health in a country. (Figueroa-Munoz et al., 2005)Health personnel include front line workers (clinicians, nurses, pharmacists, public health practitioners, medical laboratory personnel) among others as well as auxiliary personnel to the health care system. A successful and efficient health care delivery requires the right number and mix of health professionals.(Fulton et al., 2011) Further, the performance of the health care system in tackling a pandemic is closely related to the numbers, distribution, knowledge, skills and preparedness of its workforce, particularly those individuals involved in delivery of services. The involvement of health care workers in this crisis is in the whole spectrum of service delivery; tracing of suspects, quarantine of individuals who have had contact with positive cases of Covid19 care and treatment. The preparedness of the Human resource for health personnel is a key component in controlling the spread of this disease. According to the Cabinet Secretary for Health (Kenya)Front-line health workers have been trained and continue to receive updates on the characteristics of the disease as well intensive Infection Prevention and Control practices such as the use of Personal Protective Equipment as well as relevant training for all health workers. There doesn't seem to be any data on the training of the auxiliary service providers; those who are tasked with providing security, catering, and cleaning services.

\subsection{Quarantine}

Quarantine has been defined as separation of a person or persons who are not yet symptomatic but may have been exposed to a pathogen and may be at risk of developing an infection. (Use and Control, 2020). The current guidelines for preventing and controlling the spread of airborne and droplet transmitted infectious illnesses include identifying those who may have been exposed and quarantine. In the case of corona virus disease quarantine is for individuals who have had contact with a corona virus positive individual. The Centre for Disease Control (CDC) further explains that quarantine helps to prevent or stop the spread 
of disease that can occur before a person knows they are sick or if they are infected with the virus without feeling symptoms. People in quarantine during this corona virus have been advised to stay at home separating themselves from other people.(Use and Control, 2020) Quarantine can be voluntary or enforced and usually equal the length of the incubation period for the contagious disease. In the case of corona virus the quarantine is for a period of fourteen days (WHO). The government of Kenya has advised mandatory quarantine for all contacts in specified institutions.

\subsection{Role of KMTC}

Countries have come up with measures to control the spread of the disease. Country specific measures in Kenya include aggressive contact tracing, mandatory quarantine for fourteen days of all contacts and testing. All positive cases are isolated and treated at designated health facilities. The government has identified KMTC as a quarantine facility among hotels and other government institutions. Further, the training on Covid19 response and management of quarantine centers is done by the ministry of health through the country health management committee. The training is targeted at the front-line heath workers as well as the auxiliary workers. The auxiliary personnel in the college comprise of contracted service providers. It is the responsibility of the Principal of the campus to ensure that the auxiliary personnel are trained by $\mathrm{MOH}$ and prepared to be able to provide their services in the quarantine areas. The training specifies to the personnel the processes to follow while carrying out their duties to avoid contamination. For the security personnel they are trained to ensure that people getting into the compound wear masks, maintain social distance, wash their hands at the entrance and leave their contact details. The catering service provider is trained to serve food in disposable containers, leave it at the door of the room, maintain social distance and wear masks. On the other hand, the cleaning personnel should always wear masks, protective gloves and PPE if they are to get into the rooms of the clients or while handling linen from quarantined individuals. Besides frontline health care workers and managers, auxiliary health personnel are critical in the prevention of spread of the disease. This is hinged on the fact that they are interacting with quarantined individuals and as such that special attention should be paid to their role in this pandemic.

\subsection{Problem statement}

The outbreak of the corona virus started in China in December 2019. The spread of the disease is in proportions as has never been seen before with this group of the viruses. Previous outbreaks of the Severe Acute Respiratory Syndrome have not presented this magnitude of problem globally. The WHO estimates the this virus has a mortality rate of 3.4 but different countries have reported varying rates depending on the preventive strategies in place and inherent health system strengths and weaknesses.(Adams and Walls, 2020a) The WHO declared the outbreak a Public Health Emergency of International Concern on $30^{\text {th }}$ January 2020. The virus continues to spread to many countries and it is a current problem all over the world. Responsive and robust health care systems are needed in preventing any disease. A disease like the outbreak of this virus is especially a great challenge.(Gudi and Tiwari, 2020) It has caused untold strain in health systems in most countries. Governments of the world have realigned resources towards controlling its spread and mitigating its effects on the economy. Specific measures have been put such as tracing, testing treating and putting contacts of cases into quarantine.

Besides front-line health care workers and managers, auxiliary health personnel play a pivotal role in prevention of spread of the disease. These workers can also be vectors of spread if their services are not streamlined and aligned to the preventive measures put in place.

Globally, there have been reports of front- line health care workers getting infected with the corona virus disease. Reports from China estimated that 3000 health care workers got infected and at least 22 died.(Adams and Walls, 2020a) Few if any reports or studies have been done on the preparedness of auxiliary health personnel in this pandemic. Their role in preventing the spread or contributing to the spread of the disease has not been scientifically documented in our country.

1.5 Research questions

1. Which are the categories of auxiliary health personnel in the quarantine centres

2. What is the level of compliance to protocols in place for controlling the spread of Covid19 at the quarantine centers

3. How many auxiliary health personnel have contracted Covid19 at the work place?

\subsection{Objectives}

\subsubsection{Broad Objective}

The aim of this research was to determine level of compliance to Covid19 preventive protocols

\subsubsection{Specific Objectives}

i. To determine the numbers of auxiliary workers by cadre at the quarantine centers

ii. Assess the level of compliance to protocols in place for controlling the spread of Covid19 at the quarantine centers

iii. Determine the number of auxiliary workers who had contracted Covid19 at the work place

\section{METHODOLOGY}

\subsection{Study design and study site}

The study was conducted in the Kenya Medical Training College campus. It is a public middle level health training institution under the Ministry of Health. Currently the college has over fifty 71 campuses spread across the country. Most of the campuses are located next to hospital owing to the fact that most programs require clinical experience. Purposive sampling was used mainly because the sample being investigated was small (about 10 campuses) and they were only selected because they had quarantined individuals. The other 33 campuses did not meet this criterion and are thus not of interest in this research. A census of the auxiliary personnel in the selected campuses was done. An observation checklist was used

\subsection{Data collection and analysis}

An observation checklist was used to document the procedures followed by the auxiliary health personnel in providing their services. Procedures were acceptable as 'Done' when the procedures were done all the time and correctly. Skipping of a step 
or an incomplete process was classified as 'Not done'. Data analysis was carried out using univariate analysis techniques. This involved frequency distributions for categorical variables and descriptive statistics (means) for continuous variables. Categorical variables (e.g. using PPE, wearing masks, washing hands.) were presented using bar charts, pie charts and frequency distribution tables. Univariate analysis was used to determine the level of education and to give an understanding of the extend of adherence to protocols. The data was then fed into an excel sheet and then later exported to SPSS (Version 22) for analysis.

\section{RESULTS}

A total of sample of 118 auxiliary health workers in the nine campuses participated in the study. Nine key informants were interviewed. All records were complete and were eligible for analysis.

area of Deployment

Table 1. Area of deployment

\begin{tabular}{|l|l|l|}
\hline \multirow{2}{*}{ Auxiliary worker } & & \\
\hline Cleaning & Frequency & Percent \\
\hline Security & 64 & 54.2 \\
\hline Catering & 35 & 29.7 \\
\hline Total & 19 & 16.1 \\
\hline
\end{tabular}

Majority of the auxiliary workers are in cleaning at $54.2 \%$. Catering workers are the minority (18\%) of the auxiliary workers Clustered bar chart: area of deployment in relation to sex

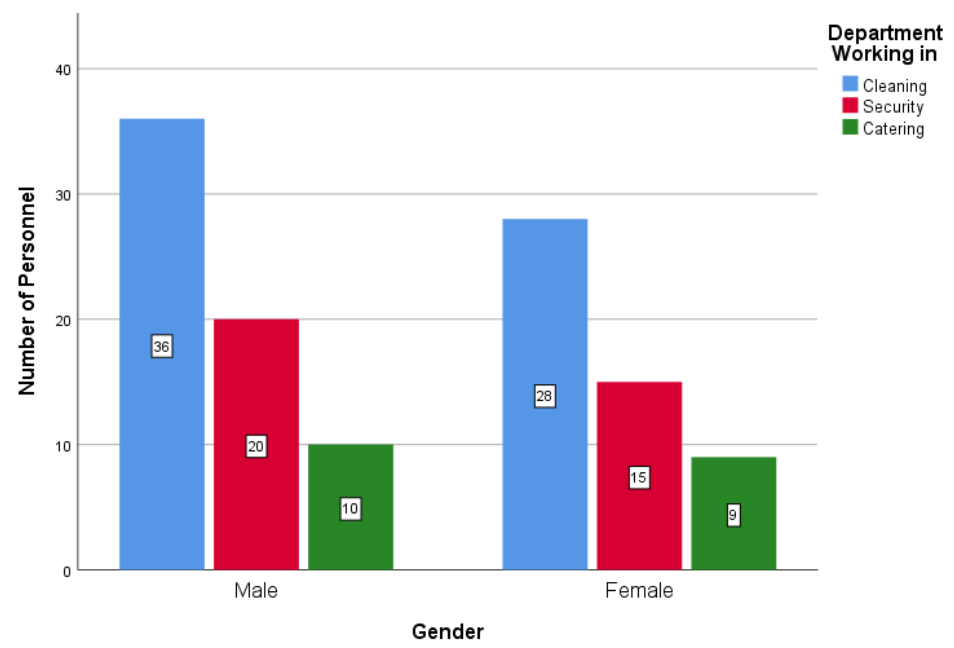

There were more male cleaners, security and catering auxiliary health workers.

\subsection{Level of education}

This study sought to know the level of education of the various auxiliary health personnel. The findings are summarized in table 2 . below:

Table 2. Level of education of auxiliary health personnel

\begin{tabular}{|l|l|l|}
\hline Level of education & Frequency & Percent \\
\hline Primary & 33 & 28.0 \\
\hline Secondary & 67 & 56.8 \\
\hline Tertiary & 18 & 15.3 \\
\hline Total & $\mathbf{1 1 8}$ & $\mathbf{1 0 0 . 0}$ \\
\hline
\end{tabular}

The majority of auxiliary workers (67) had acquired a certificate in secondary education. Very few (18\%) had proceed to college after high school.

The study further determined the relationship between level of education and area of deployment as shown below. 
Clustered bar chart: Relationship between level of education and area of deployment:

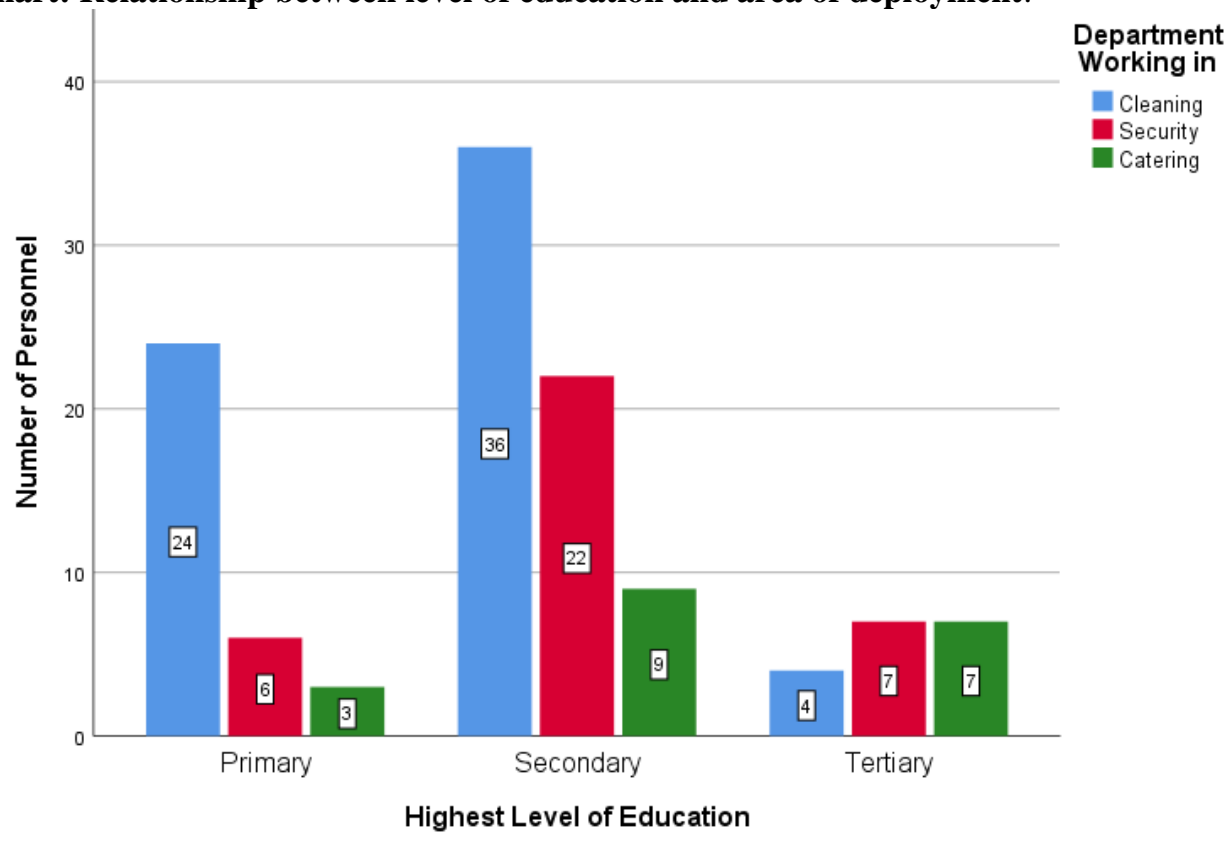

The study revealed that majority of the cleaners had primary and secondary levels of education. Only $4 \%$ had proceeded to acquire tertiary education. However, catering staff had the majority of the staff with secondary and tertiary levels of education.

\subsection{Duration at duty station}

The study sought to determine how long the auxiliary workers had been at the areas of deployment in the quarantine centres.

Table 3: Duration at area of deployment

\begin{tabular}{|l|l|l|}
\hline Duration at duty station & Frequency & Percent \\
\hline Less than One Month & 8 & 6.8 \\
\hline 1 to 4 months & 66 & 55.9 \\
\hline More than 4 months & 44 & 37.3 \\
\hline Total & 118 & 100.0 \\
\hline
\end{tabular}

The study revealed that majority of the auxiliary health workers had been deployed in the last four months. The pandemic situation had lasted less than four months hence the recent deployment. Auxiliary health personnel who had been at their areas of deployment for a period of more than four months constituted $44 \%$ of the total number.

A bar graph to demonstrate the duration of deployment was also done and is represented below

\section{Bar graph 1. Duration of deployment}




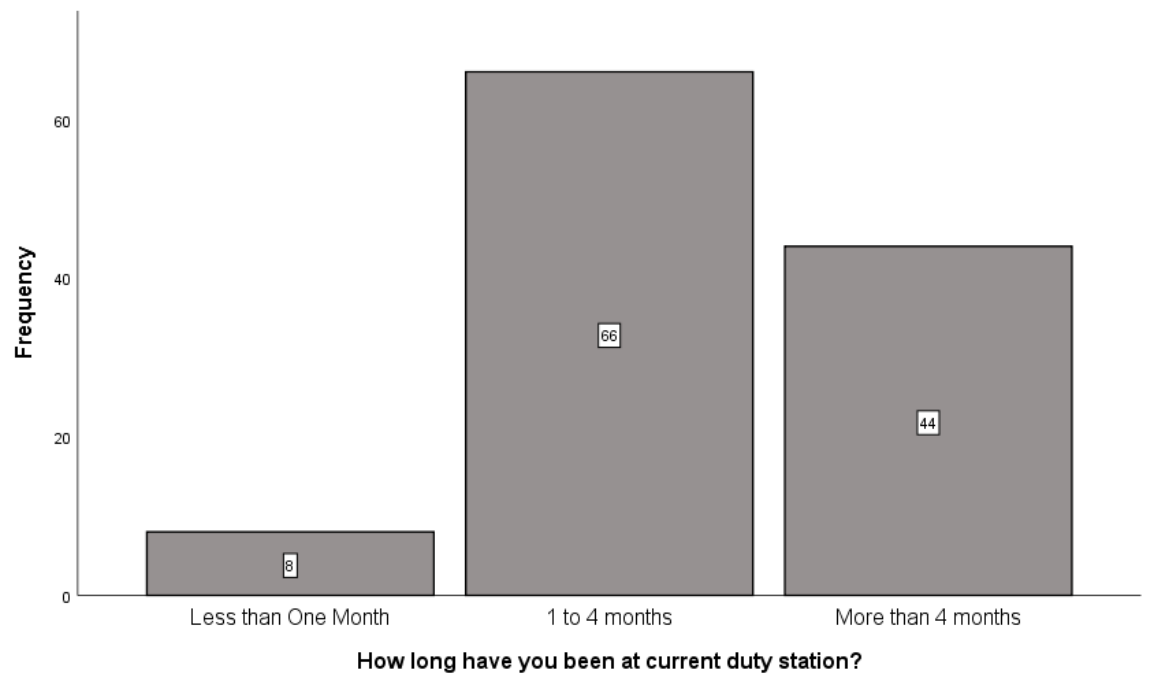

The various auxiliary workers were analyzed to determine how long they had been at their areas of deployment. This is summarized below

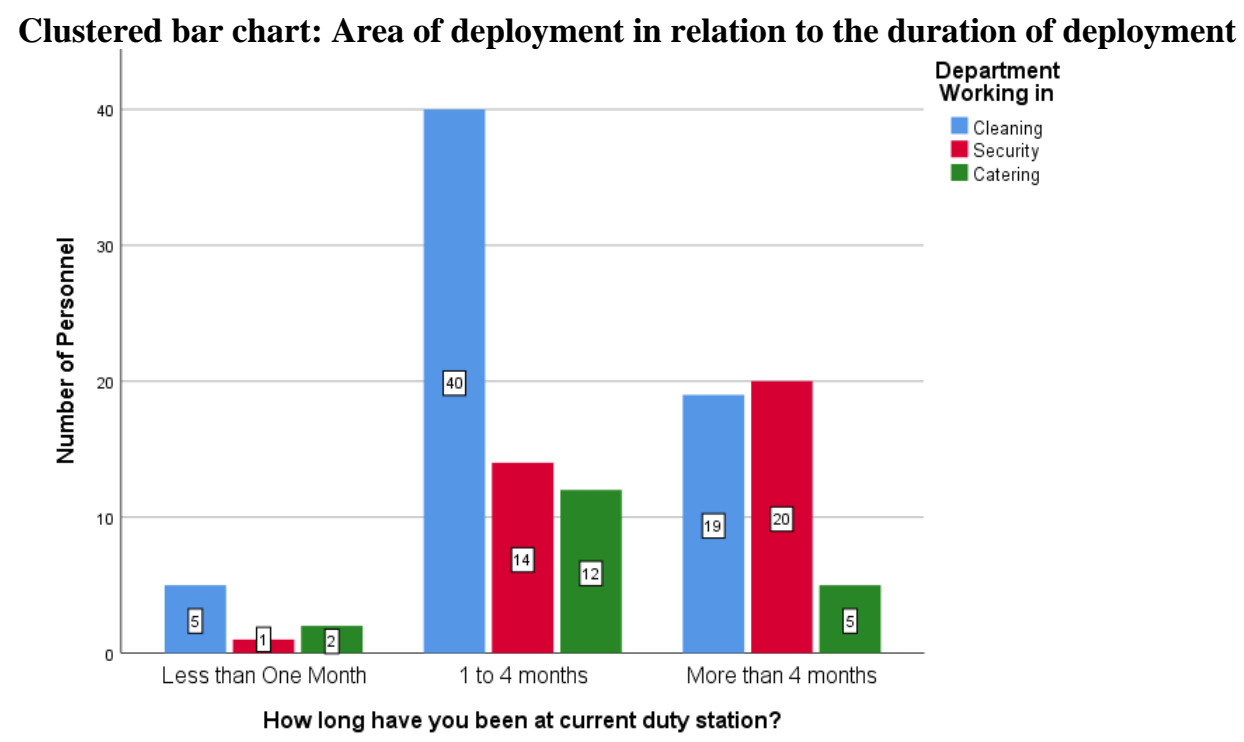

The cleaning health personnel contributed the largest population (5\%) of auxiliary health personnel who had been recently been deployed in the quarantine centres. The security personnel had been at the quarantine centres the longest.

\subsection{Knowledge on methods of spread of Covid19}

Table 4. Knowledge on how Covid19 is spread

\begin{tabular}{|l|l|l|}
\hline & & \\
& & \\
Aware how Covid 19 is spread & Frequency & Valid Percent \\
\hline Yes & 116 & 98.3 \\
\hline No & 2 & 1.7 \\
\hline Total & $\mathbf{1 1 8}$ & $\mathbf{1 0 0 . 0}$ \\
\hline
\end{tabular}

The majority of the auxiliary workers were aware of the various methods through which Covid19 is spread. They are aware that it can be spread through talking sneezing and coughing, hugging and touching contaminated surfaces. Only a small fraction (1.7\%) were not aware of the common methods of Covid19 spread.

The study sought to find out if there was a difference in the awareness in the different cadres. 
The following clustered bar chart represents the findings:

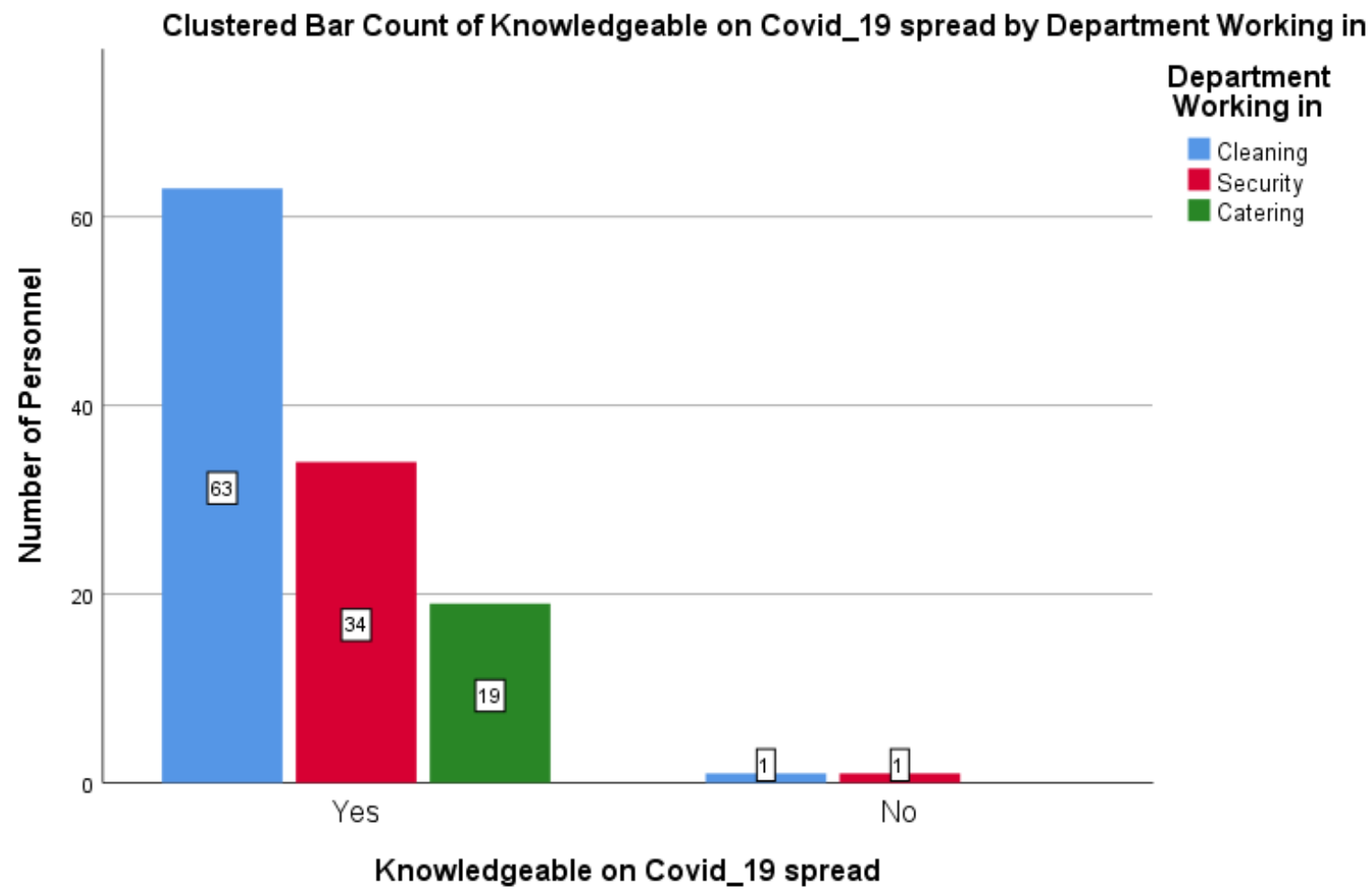

The various auxiliary workers had good knowledge on the methods of spread of Covid 19.

It is important to note that all the catering staff were aware of the ways through which the virus is spread. The proportion of security and cleaning staff who did not know how Covid19 is spread was equal.

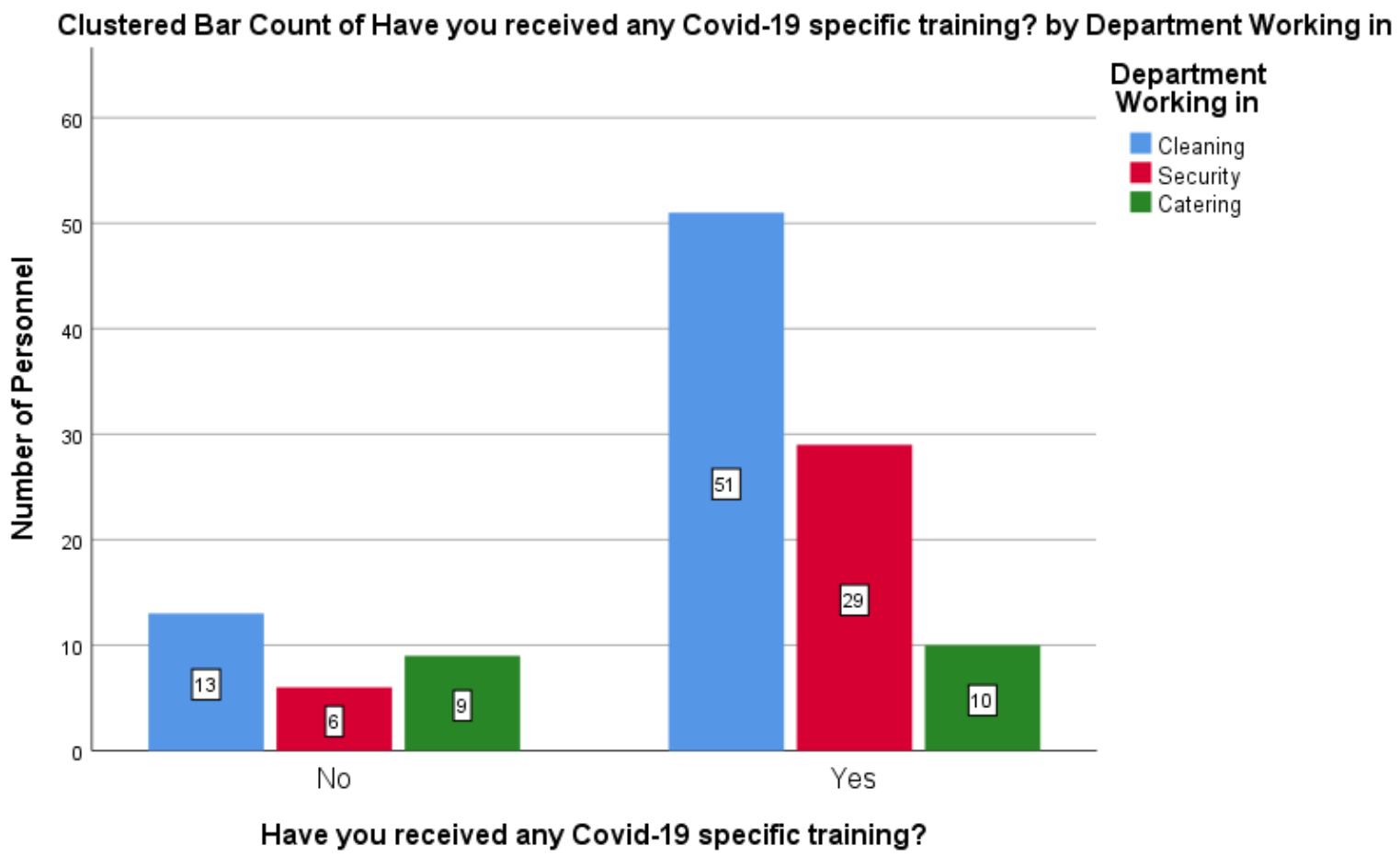

The majority of the auxiliary health personnel had received the training in readiness to work in the quarantine centres. The proportion of catering staff who had received the training (10\%) was almost equal to the fraction that had not received the training $(9 \%)$. 


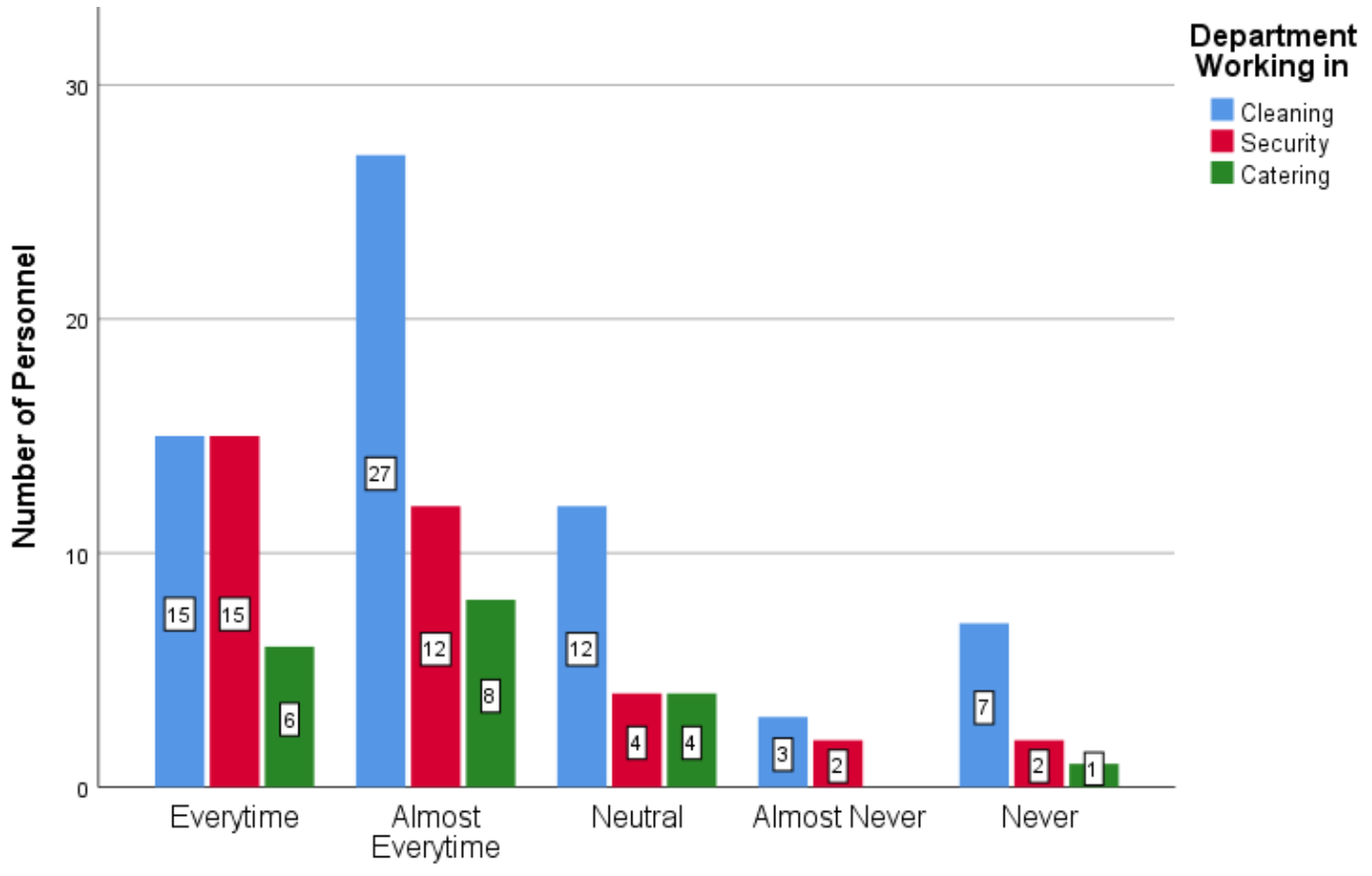

Do you consider yourself at risk of contracting Covid-19 while at ...

All the auxiliary health personnel considered themselves at risk of contracting Covid19 at the quarantine centres. The cleaning workers considered themselves at the most risk of contracting Covid19.

\subsection{Adherence to Infection Control protocols}

The quarantine centres had put protocols in place such as wearing of face masks, hand washing, using of alcoholbased sanitizer, keeping social distance, wearing PPE specifically hazmat suits, boots, googles and gloves. The adherence to these protocols was evaluated by observation of the auxiliary staff while at work using an observation checklist and the findings are summarized below.

Table 5: Security personnel

\begin{tabular}{|c|c|c|c|}
\hline Variable & & Frequency & Percent \\
\hline \multicolumn{4}{|c|}{ Maintains social distance } \\
\hline & No & 2 & 22.2 \\
\hline & Yes & 7 & 77.8 \\
\hline \multicolumn{4}{|c|}{ Wears mask } \\
\hline & Yes & 9 & 100.0 \\
\hline \multicolumn{4}{|c|}{ Washes hands/uses hand rub } \\
\hline & & 9 & 100 \\
\hline \multicolumn{4}{|c|}{$\begin{array}{l}\text { Guides Visitor to wash hands or use } \\
\text { hand rub }\end{array}$} \\
\hline & No & 6 & 66.7 \\
\hline & Yes & 3 & 33.3 \\
\hline \multicolumn{4}{|c|}{ Asks visitor to wear face mask } \\
\hline & No & 4 & 44.4 \\
\hline & Yes & 5 & 55.6 \\
\hline
\end{tabular}

The security personnel were compliant in most of the personal aspects of Covid19 prevention measures. They are tasked with ensuring all people getting into the institutions are following the guidelines in place for prevention of Covid19 specifically wearing of masks and guiding visitors to wash hands or use a hand rub as well as wearing masks. The analysis of this practices is indicated in the figures below 
Figure 1. Security personnel asks visitor to wear masks

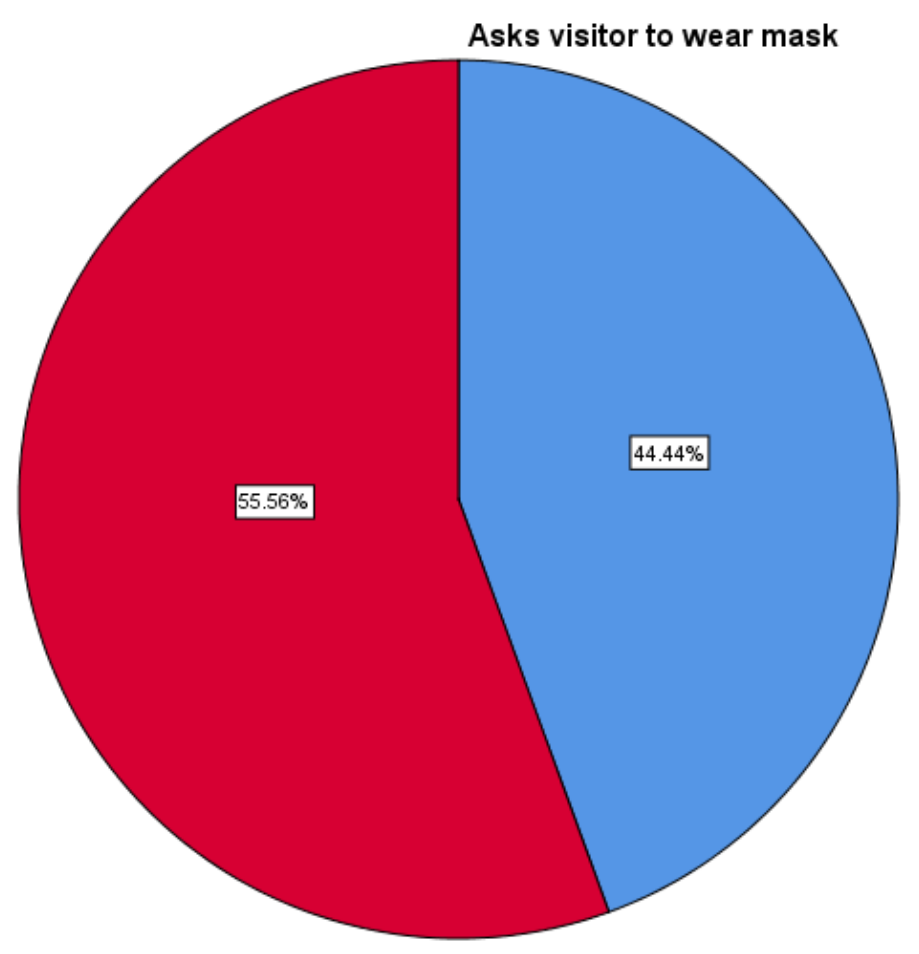

It is notable that $44 \%$ of the cases visitors were not asked to wear while entering the institution which is a lapse in the Covid 19 prevention guidelines

Figure 2. Security personnel guides visitors to wash hands or use a hand rub

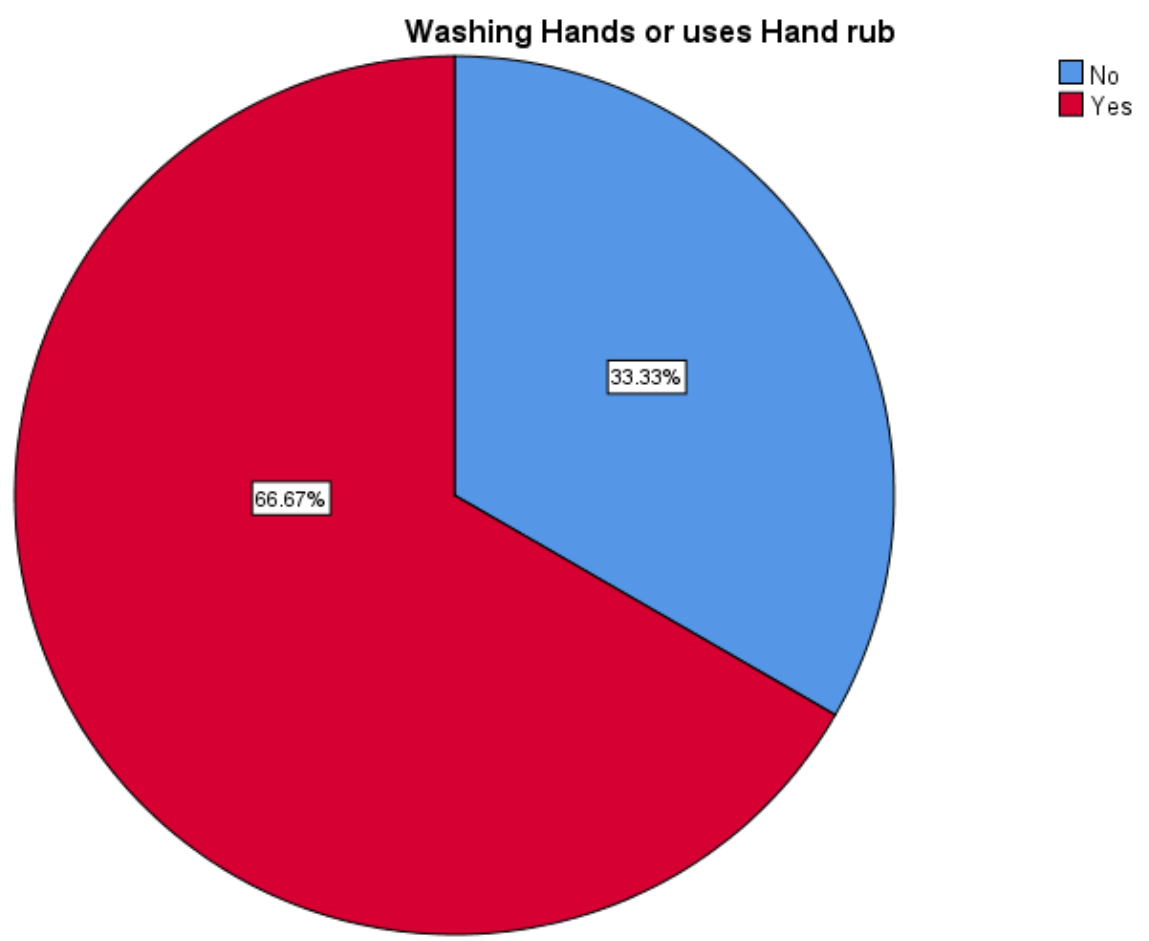

From the observation, security personnel guided visitors to sanitize their hands through hand washing or providing a hand rub in $66.6 \%$ of the cases. However, in $33.3 \%$ of the cases visitors were not asked to wash hands or use an alcohol -based hand rub. This is also a lapse in the effort of the institution to prevent the spread of Covid19. 
Table 6: Cleaning

\begin{tabular}{|l|l|l|}
\hline Variable & Frequency & Percent \\
\hline Maintains social distance & & \\
\hline No & 4 & 44.4 \\
\hline Yes & 5 & 55.6 \\
\hline Wears PPE & & \\
\hline No & 4 & 44.4 \\
\hline Yes & 5 & 55.6 \\
\hline $\begin{array}{l}\text { Uses designated Waste separation } \\
\text { process }\end{array}$ & & \\
\hline No & 1 & 11.1 \\
\hline Yes & 8 & 88.9 \\
\hline Has appropriate cleaning tools & & \\
\hline No & 2 & 22.2 \\
\hline Yes & 7 & 77.8 \\
\hline Washes hands/uses hand rub & & \\
\hline & 9 & 100 \\
\hline
\end{tabular}

The cleaning personnel come into direct surfaces touched by the quarantine clients while cleaning. Therefore, the wearing of masks and PPE are critical for them. It is notable that there was a lapse in all the protective measures in place i.e maintaining social distance amongst themselves, not wearing PPE, proper waste disposal, using the appropriate tools to clean and handwashing.

At the time of this study there were three (3) auxiliary personnel who had contracted covid 19 and they were all cleaners.

Table 7: Catering personnel

\begin{tabular}{|l|l|l|}
\hline Variable & Frequency & Percent \\
\hline Maintains social distance & & \\
\hline No & 4 & 44.4 \\
\hline Yes & 5 & 55.6 \\
\hline Serves food using disposable containers & 4 & \\
\hline No & 5 & 44.4 \\
\hline Yes & 5 & 55.6 \\
\hline Wears mask & 2 & \\
\hline No & 7 & 22.2 \\
\hline Yes & & 77.8 \\
\hline Washes hands & 3 & \\
\hline No & 6 & 33.3 \\
\hline Yes & & 66.7 \\
\hline Maintains social distance with clients & 2 & \\
\hline No & 2 & 22.2 \\
\hline Yes & 7 & 77.8 \\
\hline
\end{tabular}

The catering personnel were observed to have lapses in all areas of prevention aspects. They were observed to wear mask in $77.8 \%$ of the cases but in $22.2 \%$ of the cases they did not. They were observed to put themselves at risk by not maintaining social distance while serving clients in $22.2 \%$ of the cases. This is a major lapse in the protocols in place for preventing Covid 19.

\subsection{Covid19 Positive Auxiliary Health Personnel}

The study sought to determine the number of auxiliary health personnel who had contracted Covid19 by the time of the study 
Table 8: Covid19 Positive Auxiliary Health Personnel

\begin{tabular}{|l|l|}
\hline & \\
& \\
\hline Category & Frequency \\
\hline Security & 0 \\
\hline Cleaning & 3 \\
\hline Catering & 0 \\
\hline Total & $\mathbf{3}$ \\
\hline
\end{tabular}

At the time of this study there were three (3) auxiliary personnel who had contracted Covid19 and they were all cleaners.

\section{DISCUSSION AND CONCLUSION}

The study determined that there were auxiliary personnel in the key areas at the quarantine centres. The study revealed lapses in adherence all areas of the Covid19 prevention protocols.

The WHO emphasizes hygiene especially hand hygiene as one of the strategies of combating Covid19. In quarantine centres, the cleaning staff are required to follow the protocols correctly and consistently (WHO, 2020). The WHO gives guidelines on how to maintain a clean environment in the quarantine centres. The cleaners are encouraged to increase the frequency of cleaning of commonly touched surfaces such as light switches, countertops, chair arms, escalator railings, elevator buttons, doorknobs, and handles. (Adams and Walls, 2020b)

The Covid19 prevention protocols include handwashing, wearing PPE for health workers, wearing a face mask maintaining social distance and use of an alcoholbased sanitizer. One of the recommendations of the WHO is frequent hand washing with soap. This non pharmaceutical intervention for the control of Covid19 has been found to have significant protective effects. (Benkouiten, Brouqui and Gautret, 2014). In a communal residence, the use of hand rub was shown to reduce symptoms of SARS in a communal student residence by $40 \%$. (Javier et al., 2012). A combination of the use of hand rub and hand washing was also shown to reduce illness absenteeism in a private elementary school by $41.9 \%$. (Javier et al., 2012). The WHO has recommended handwashing and or the use of hand rub as one of the key ways of keeping Covid19 at bay in the community. These interventions are prescribed for everyone during this Covid19 pandemic. Auxiliary workers working in quarantine areas are already at risk since quarantined individuals are contacts of positive cases. There is the need to follow the laid down protocols. Wearing of PPE for health care personnel has been taunted as critical in the prevention of Covid19. The availability of the same to healthcare workers is a priority during this pandemic. (Adams and Walls, 2020b).Healthcare workers use personal protective equipment to shield themselves from droplets from coughs, sneezes or other body fluids from infected patients and contaminated surfaces that might infect them. PPE may include aprons, gowns or coveralls (a one-piece suit), gloves, masks and breathing equipment (respirators), and goggles. The WHO has also prescribed the use face masks during this pandemic. The auxiliary workers were found not to adhere to this recommendation effectively. In a study conducted to check the effectiveness of face masks or google among health care workers visiting and caring for children aged up to five with respiratory syncytial virus and symptoms of respiratory disease was effective ( $5 \%$ illness rate in google wearers against $61 \%$ in no google controls. (Javier et al., 2012). Adherence to guidelines such as CDC guidelines enhances the safety of healthcare professionals(Adams and Walls, 2020b). during this pandemic in Wuhan China, many other HCP from other provinces were not infected due to the use of PPE supporting the importance of carefully protecting health care workers in the outbreak of a high transmissible infectious disease(Pan et al., 2020)

In conclusion, the MOH response to the Covid19 including the quarantine of contacts of positive cases was aimed at reducing the spread of the disease within the community. While there are many strategies of ensuring that health workers follow prevention guidelines as well have the appropriate PPE, there is little focus on the auxiliary workers. There is need for strengthened enforcement of protocols by these workers.

\section{Ethical consideration}

Ethical approval was obtained from the KMTC Ethics Review Committee. Only participants who consented to participate were included in the study.

\section{Competing interests}

Authors declare no competing interests

\section{Funding}

Funding was received from the Kenya Medical Training College

\section{ACKNOWLEDGEMENT}

Kenya Medical Training college, auxiliary health personnel who participated in the study.

\section{REFERENCES}

[1] Adams, J. G. and Walls, R. M. (2020a) 'Supporting the Health Care Workforce during the COVID-19 Global Epidemic', JAMA - Journal of the American Medical Association, 60611, pp. 2-3. doi 10.1001/jama.2020.3972.

[2] Adams, J. G. and Walls, R. M. (2020b) 'Supporting the Health Care Workforce during the COVID-19 Global Epidemic', JAMA - Journal of the American Medical Association, 323(15), pp. 1439-1440. doi 10.1001/jama.2020.3972. 
[3] Benkouiten, S., Brouqui, P. and Gautret, P. (2014) 'Non-pharmaceutical interventions for the prevention of respiratory tract infections during Hajj pilgrimage', Travel Medicine and Infectious Disease, 12(5), pp. 429-442. doi: 10.1016/j.tmaid.2014.06.005.

[4] Chang, A. et al. (2020) 'Cleaning and Disinfectant Chemical Exposures and Temporal Associations with COVID-19 - National Poison Data System, United States, January 1, 2020-March 31, 2020', MMWR. Morbidity and Mortality Weekly Report, 69(16), pp. 496-498. doi: 10.15585/mmwr.mm6916e1.

[5] Figueroa-Munoz, J. et al. (2005) 'The health workforce crisis in TB control: a report from high-burden countries.', Human resources for health, 3(1), p. 2. doi: 10.1186/1478-4491-3-2.

[6] Fulton, B. D. et al. (2011) 'Health workforce skill mix and task shifting in low income countries: a review of recent evidence.', Human resources for health. BioMed Central Ltd, 9(1), p. 1. doi: 10.1186/1478-4491-9-1.

[7] Gudi, S. K. and Tiwari, K. K. (2020) 'Preparedness and lessons learned from the novel coronavirus disease', International Journal of Occupational and Environmental Medicine, 11(2), pp. 108-112. doi: 10.34172/ijoem.2020.1977.

[8] Javier, F. et al. (2012) 'Main results Authors ' conclusions', Cochrane Library, 6(November 2017), pp. 1-54. doi: 10.1002/14651858.CD003769.pub4/full.

[9] Lai, T. H. T. et al. (2020) 'Stepping up infection control measures in ophthalmology during the novel coronavirus outbreak: an experience from Hong Kong', Graefe's Archive for Clinical and Experimental Ophthalmology, 258(5), pp. 1049-1055. doi: 10.1007/s00417-020-04641-8.

[10] Mason, D. J. and Friese, C. R. (2020) 'Protecting Health Care Workers Against COVID-19 - and Being Prepared for Future Pandemics', JAMA Health Forum, 1(3), p. e200353. doi: 10.1001/jamahealthforum.2020.0353.

[11] Pan, A. et al. (2020) 'Association of Public Health Interventions with the Epidemiology of the COVID-19 Outbreak in Wuhan, China', JAMA Journal of the American Medical Association, 323(19), pp. 1915-1923. doi: 10.1001/jama.2020.6130.

[12] 'The effectiveness of quarantine and isolation determine the trend of the COVID-19 epidemics in the $\mathrm{f}^{\prime}$ (no date).
[13] Use, E. and Control, D. (2020) 'Isolation Precautions: Quarantine for Pediatric Patients ( Home Health Care ) - CE Isolation Precautions : Quarantine for Pediatric Patients ( Home Health Care ) - CE', pp. 1-5.

[14] WHO (2020) 'Considerations for quarantine of individuals in the context of containment for coronavirus disease ( COVID-19)', Who, (February), pp. 3

\section{AUTHORS}

First Author_Jacinta Mukonzo, Kenya Medical Training College B. Pharm, MPH, jmukonzo@ kmatc.ak.ke or Jacinta.mukonzo@gmail.com, Participated in proposal development, data collection and analysis, manuscript writing Second Author: Marsellah Ogendo, Kenya Medical Training College, B Pharm, MSc (Health Professions Education) mogendo@kmtc.ac.ke, Participated in proposal development and data collection

Third Author: Kelly Oluoch, Kenya Medical Training Colllege, B. Pharm, MBA, koluoch@ kmct.ak.ke, Participated in data collection

Fourth Author: Eglah Kiplagat, Kenya Medical Training College,ekiplagat@kmtc.ac.ke, Participated in data collection Fifth Author: Claris Ambale, Machakos Level 5 Hospital, B. Phar, Mpharm, clarisambale1983@gmail.com, Participated in manuscript writing

Corresponding Author : Jacinta Mukonzo, jmukonzo@kmtc.ac.ke or Jacinta.mukonzo@gmail.com 